\title{
Research on Ultrasonic Testing Technology of Gas Leakage in Aircraft Air-Tightness System
}

\author{
Yin Dawei ${ }^{*}, 1,2$ and Lin Jun ${ }^{1}$ \\ ${ }^{I}$ College of Instrumentation \& Electrical Engineering, Jilin University, Changchun 130026, P.R. China \\ ${ }^{2}$ Aircraft System Staff Room, Aviation University of Air Force, Changchun 130022, P.R. China
}

\begin{abstract}
Gas leakage testing of aircraft air-tightness system has always been an important work in the aircraft maintenance. Quantitative testing of gas leakage signal drowned in noise is hardly realized alone by ultrasonic detection instrument. In this paper, theory of ultrasonic and small orifice gas leakage has been employed, and a mathematical model based on ultrasonic testing gas leakage of aircraft air-tightness system has been deduced. Through improved chaos Duffing oscillator equation and improved wavelet threshold denoising algorithm, weak period signal in the strong noise background has been successfully distinguished and denoised. Along with software and hardware assistant discrimination means, ultrasonic testing will be more effective to stop gas leakage of aircraft air-tightness system.
\end{abstract}

Keywords: Duffing oscillator equation, Gas leakage, Ultrasonic testing, Wavelet threshold denoising, Air Tightness, Maintenance.

\section{INTRODUCTION}

Aircraft is a typical aviation machine manufactured by people, which is complex and is widely used in modern society, bearing a series of missions like traffic transport and homeland defense. Aiming to aircraft air-tightness system, periodical or emergency testing work should be done to ensurepassengers' safety and normal flight.

Traditional testing technology on gas leakage of airtightness system includes water test method, applying soap liquid method, stethoscope method, flow measurement method, chemical method, absolute pressure method, differential pressure method and Helium measurement method . $[1,2]$, all of them have obvious defects, like backward testing mode, lower testing accuracy, ponderous testing equipment, and non-online testing.

At present, as a kind of widespread NDT technology, ultrasonic testing method has already overcome the defects of traditional testing methods, and many testing instruments on air-tightness system have successfully been developed. U1trasonic testing instruments applied to aircraft air-tightness system, which are mainly based on qualitative testing and only search leakage position, have not built applicative mathematical model having a concrete leakage degree to a certain point. On the other hand, in gas leakage signals in the strong noise background, software assistant denoising and analysis should be necessarily developed, because hardware instrument cannot alone eliminate the strong noise interference.

\footnotetext{
*Address correspondence to this author at the College of Instrumentation \& Electrical Engineering, Jilin University, Changchun, Jilin, 130022, P.R. China; Tel: +86 15526865341; E-mail: effortman0434@163.com
}

Consequently, in the paper, above-mentioned questions are discussed. As a result of research, a mathematical model based on ultrasonic testing of gas leakage in aircraft airtightness system is built. By means of improved chaos Duffing oscillator equation and improved wavelet threshold denoising algorithm, gas leakage signal characteristics in the strong noise background were analyzed. The research will help ultrasonic testing technology to better serve for aircraft maintenance.

\section{BUILDING MATHEMATICAL MODEL FOR GAS LEAKAGE OF AIRCRAFT AIR-TIGHTNESS SYS- TEM}

Gas leakage of aircraft air-tightness system has characteristics coincident with ultrasonic $40 \mathrm{KHz}$ frequency gas leakage property of small orifice [3].

Aircraft air-tightness system includes oxygen and compressed air system with high pressure property, and passengers' air-tightness cockpit with low pressure property. In oxygen and compressed air system, gas leakage phenomenon appears with high pressure gas leakage in the form of small orifice. In air-tightness cockpit, low pressure gas leakage in the form of small orifice is common, because human body possesses less ability to bear pressure and differential pressure inside and outside cockpit [4-6].

A well-known scholar Ma Da-you emphasized that $[7,8]$, ultrasonic is produced by turbulence and impulsive noise ; the formula of ultrasonic sound pressure level can be deduced on the basis of gas leakage orifice dimension and system pressure change. That is,

$$
L=80+20 \log \frac{D}{D_{0}}+10 \log \frac{\left(p-p_{0}\right)^{4}}{p_{0}^{2}\left(p-0.5 p_{0}\right)^{2}}
$$

In formula $1, L$ represents the sound pressure level one meter away from gas leakage orifice in the vertical direc- 
tion; $D$ represents the gas leakage orifice diameter $\left(D_{0}=1 \mathrm{~mm}\right) ; p_{0}$ represents the absolute pressure environment atmosphere; $p$ represents the position of pressure of gas leakage orifice.

On account of larger difference between gas leakage rate of small orifice and the volume of air-tightness system, in aircraft air-tightness system, gas pressure changes slowly and gas leakage rate keeps constant to a certain period. When air pressure inside and outside the air-tightness system is stable, gas flow velocity of leakage orifice becomes stable. So, Formula 2 may be required to describe the relation between gas flow velocity and air pressure inside the system.

$$
\left\{\begin{array}{l}
V=K P \psi(\sigma) / \sqrt{R T_{1}} \\
\psi(\sigma)= \begin{cases}\sqrt{\sigma^{2 / k}-\sigma^{(k+1) / k}} & 0.528<\sigma<1 \\
0.2588 & 0<\sigma<0.528\end{cases}
\end{array}\right.
$$

In Formula 2, $V$ represents the gas flow velocity; $T_{1}$ represents the absolute temperature; $\sigma=p_{0} / p, p_{0}$ and $p$ have the same meaning with that of Formula $1 ; R$ represents gas constant; $K=\sqrt{2 k /(k-1)}$, to air, $k=1.4$, then $K=2.646$.

When gas leakage signal of air-tightness system is required by the ultrasonic sensor, the corresponding sound pressure level $L$ of gas leakage will be obtained. According to Formula 1, average diameter of orifice can be computed by Formula 3 .

$$
D=D_{0} * 10 \frac{L-80-10 \log \frac{\left(p-p_{0}\right)^{4}}{p_{0}^{2}\left(p-0.5 p_{0}\right)^{2}}}{20}
$$

In Formula 3, due to subcritical flow characteristics of small orifice gas leakage in the aircraft air-tightness system, $0.528<\sigma<1$ is required and formula 4 will be deduced.

$V=K p \sqrt{\sigma^{\frac{2}{k}}-\sigma^{\frac{k+1}{k}}} / \sqrt{R T_{1}}$

When orifice mechanics average diameter $D$ and gas flow velocity $V$ are known, Reynolds number $R_{e}$ will be obtained.

$R_{e}=\frac{\rho V D}{\mu}=\frac{\rho V A_{e}}{\mu A_{e}}=\frac{q_{m} D}{\mu \mu_{1} \frac{\pi}{4} D^{2}}=\frac{4 q_{m}}{\mu \mu_{1} \pi D}$

In Formula 5, $\mu$ represents gas dynamic viscosity; $\mu_{1}$ represents flow rate coefficient, $\mu_{1}<1$, its value is confirmed by experiment; $A_{e}$ represents the effective small orifice area; $q_{m}$ represents gas quality flow rate, i.e. gas leakage quantity of small orifice.

At this time, by combining formula 3,4 , and 5 , a mathematical model based on gas leakage of aircraft air-tightness system can be obtained. That is,

$$
\begin{aligned}
& q_{m}=\mu_{1} \pi \frac{\rho V D^{2}}{4}=\frac{\mu \mu_{1} \pi}{4} * \operatorname{Re} * D_{0} * 10 \frac{\frac{L-80-10 \log \frac{\left(p-p_{0}\right)^{4}}{p_{0}{ }^{2}\left(p-0.5 p_{0}\right)^{2}}}{20}}{\rho \frac{K p \sqrt{\sigma^{\frac{2}{k}}-\sigma^{\frac{k+1}{k}}}}{\sqrt{R T_{1}}} * D_{0}{ }^{2} * 10 \frac{\frac{L-80-10 \log \frac{\left(p-p_{0}\right)^{4}}{p_{0}{ }^{2}\left(p-0.5 p_{0}\right)^{2}}}{10}}{4}} \\
& =\mu_{1} \pi \frac{{ }^{2}}{4}
\end{aligned}
$$

In gas leakage degree evaluation of aircraft air-tightness system, inside and outside pressure value of air-tightness system, flow rate, and gas temperature at the gas leakage orifice, all these parameters can be gained by measurement or formula transformation. Formula 6 indicates physical parameters like gas leakage rate of small orifice and orifice mechanics average diameter; therefore, sound pressure level of $40 \mathrm{KHz}$ frequency point is tested to a certain distance of gas leakage orifice, and inside and outside pressure of airtightness system is also measured.

Therefore, according to Formula 6, a mathematical model based on ultrasonic testing gas leakage of aircraft airtightness system will be built, which has better practicability.

\section{IMPROVEMENT ANALYSIS OF TESTING METH- OD OF WEAK PERIOD SIGNAL}

At present, maintenance works of all kinds of aircrafts are developed at tarmac of airport or hanger. Numerous electron equipments are used, and many testing works are carried out. Electromagnetic interference phenomenon is obvious around maintenance field, due to which gas leakage testing effect is badly influenced. Under the background of strong noise, gas leakage signal testing of aircraft air-tightness system belongs to typical weak period signal testing. By use of chaos theory and wavelet denoising analysis, discrimination and denoising of weak period signal in the strong noise background will be effectively developed [9-12].

\subsection{Improved Chaos Duffing Oscillator Equation}

Traditional Duffing oscillator equation is a better means to distinguish weak period signal. But, this equation is sensitive to statistical property of oscillator system, strong noise, signal characteristics, and cannot accurately analyze signal property. So, many scientific researchers do plenty of works to improve Duffing oscillator equation.

In the paper, considering nonlinear recovery force characteristic of chaos system, as well as its obvious influence on high frequency weak signal, nonlinear recovery force item of traditional Duffing oscillator equation will be changed into $\alpha x^{5}(t)-\beta x^{7}(t)$, meanwhile, $\alpha=1, \beta=1$. Adding weak period signal including noise into chaos system, expression of Duffing oscillator equation will be changed into,

$$
\frac{1}{\omega^{2}} \ddot{x}(t)+\frac{k}{\omega} \dot{x}(t)+\alpha x^{5}(t)-\beta x^{7}(t)=\gamma_{d} \cos \omega t+a \cos (\omega t)+n(t)
$$

Or it is written as,

$$
\left\{\begin{array}{l}
\dot{x}=\omega y \\
\dot{y}=\omega\left(-k y+a x^{5}-\beta x^{7}+\gamma_{d} \cos (\omega t)+a \cos (\omega t)+n(t)\right)
\end{array}\right.
$$

In Formula $7, \dot{x}$ and $\dot{y}$ represent state variable; $k$ represents damp ratio; $\gamma_{d}$ represents chaos threshold; $\omega$ represents driving force frequency; $a \cos (\omega t)+n(t)$ represents including noise weak period signal, $n(t)$ represents noise.

Comparison on weak period signal discrimination of different Duffing oscillator equations in a strong noise background is given. 


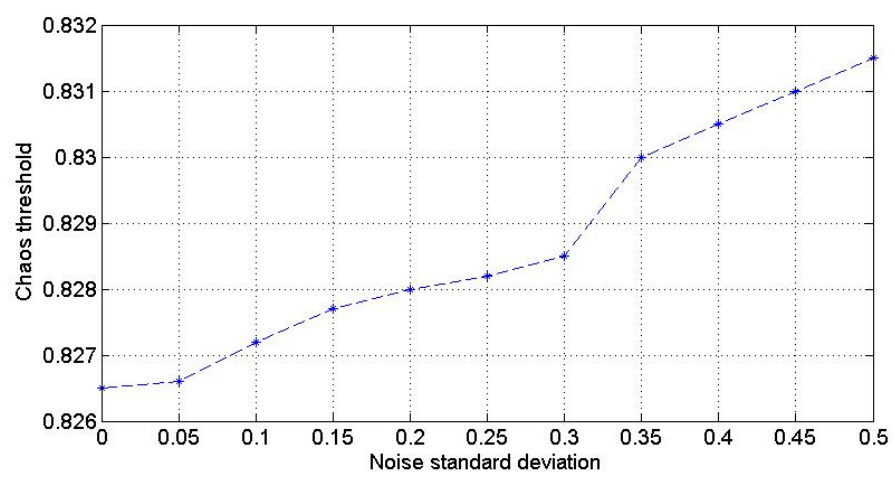

(a). Traditional method

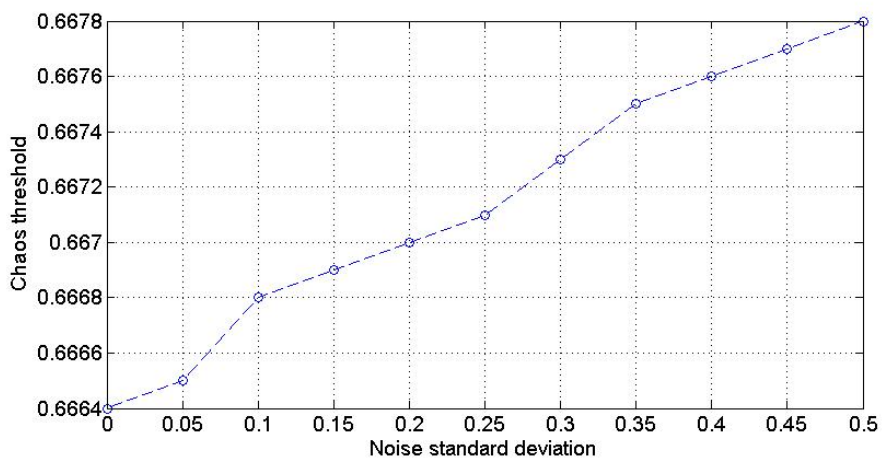

(b). Improved method in the paper

Fig. (1). Relational graph on chaos threshold of two kinds of chaos systems changing with noise standard deviation.

Aiming to traditional Duffing oscillator equation and improved Duffing oscillator equation in the paper, Gaussian white noise with zero mean value and variable noise standard deviation are combined into a chaos system. Noise standard deviation is changed with 0.05 interval in the range from 0 to 0.5 . The variable states of chaos threshold with different noise standard deviation are shown in Fig. (1).

Fig. (1) indicates that when noise standard deviation is lesser, chaos thresholds of two kinds of chaos systems change little. However, when noise standard deviation increases up to a certain degree, chaos thresholds of two kinds of chaos systems change significantly. By contrast, in the same variable range of noise standard deviation, chaos threshold of traditional chaos system changes more obviously than that of the improved chaos system in the paper. Document 13 emphasized that noise variation influences the critical state of chaos system, and even changes critical state of chaos system. That is to say, with the strengthening of noise, measurement error of weak period signal will constantly increase. In comparison with traditional chaos system, chaos threshold of improved chaos system in the paper changed in a smaller range. Consequently, improved chaos system in the paper is a better means to test weak period signal under the background of strong noise.

Two, testing capacity analysis on high frequency weak period signal.

Separately, traditional Duffing oscillator equation and improved Duffing oscillator equation were added in the weak period signal $0.0006 * \cos (40000 * t)$ including Gauss- ian white noise with zero mean value and 0 or 0.15 noise standard deviation, and then phase track states of chaos system were observed. The results are shown in the Fig. (2).

Fig. (2) reveals that for the same chaos threshold, when noise standard deviation is zero, phase tracks required by the two kinds of methods both enter large scale period state. When noise standard deviation is 0.15 , in the traditional method, phase track of chaos system returns to the chaos state and cannot be used to test the weak period signal, because chaos critical threshold changes much more than the original state under the influence of strong noise. Nevertheless, in the improved method, phase track of chaos system maintains large scale period state, with the capacity on testing weak period signal being still useful, because chaos critical threshold changes in a small and effective range under the influence of strong noise.

The above-mentioned analysis indicates that with continuous increase in the noise, with respect to different chaos systems, the capacity of testing weak period signal will be different. On one hand, noise intensity becomes greater, making the nonlinear characteristic of chaos system more obvious. On the other hand, with period driving force frequency changing from low frequency to high frequency, nonlinear characteristic of chaos system is strengthened to some certain degree. Hence, Duffing oscillator equation with high order nonlinear recovery force item will satisfy nonlinear characteristic demand of chaos system, and is better to distinguish high frequency weak period signal in the strong noise backgroud. 

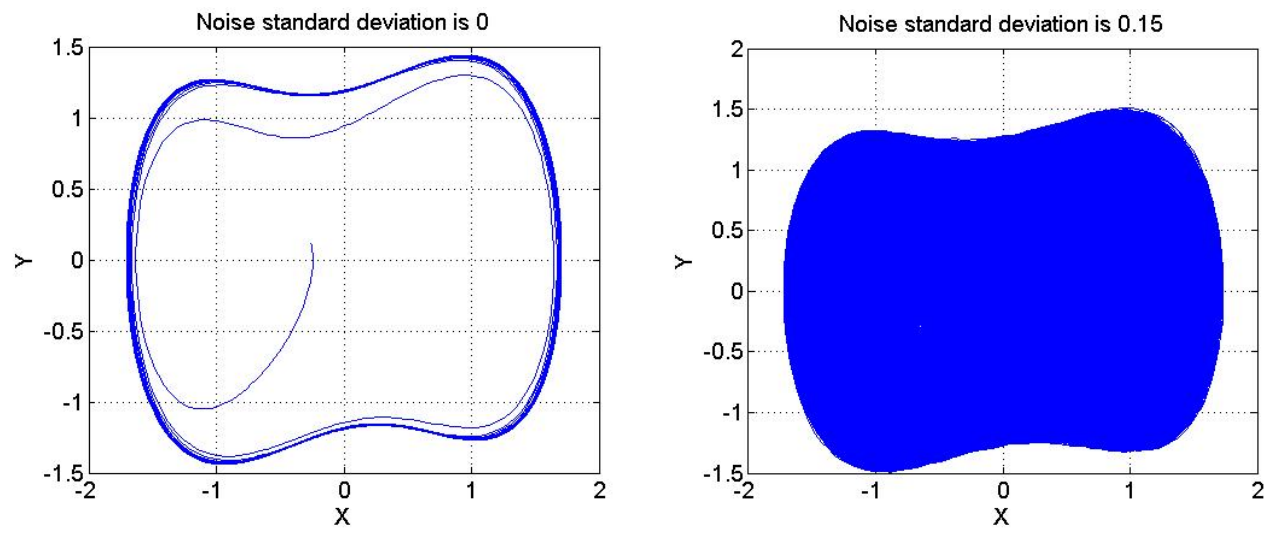

(a). Traditional method
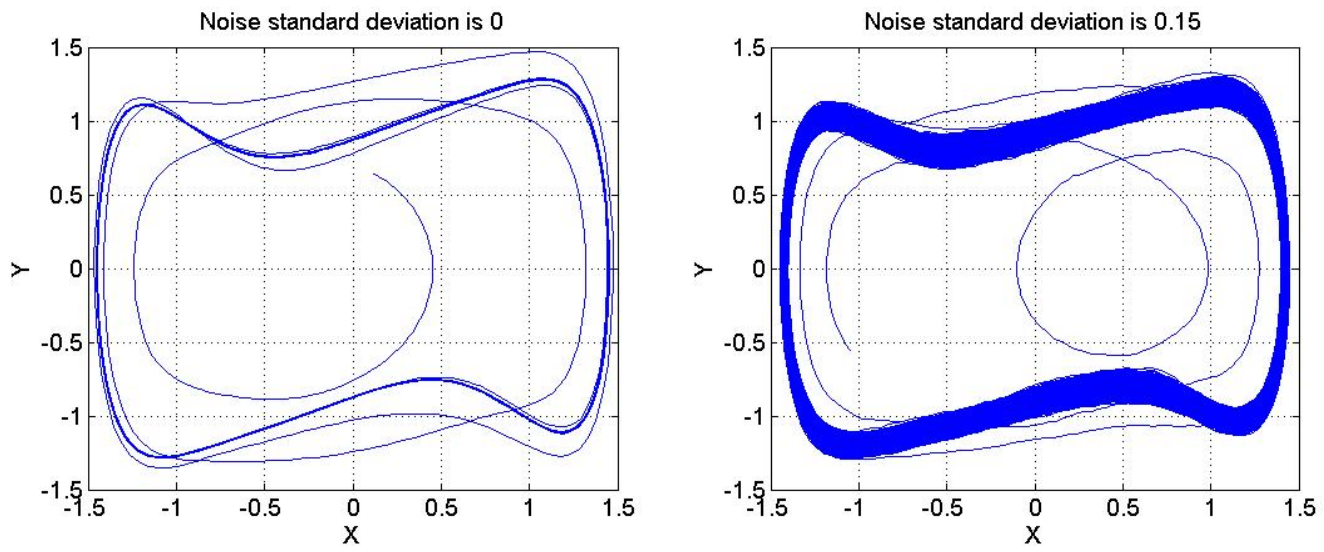

(b). Improved Method in The Paper

Fig. (2). Phase track diagrams of two kinds of chaos systems, when noise standard deviation is 0 and 0.15 . (a. Traditional method; b. Improved method in the paper).

\subsection{A New Type of Wavelet Threshold Denoising Method}

In Donoho soft or hard threshold denoising method [1316], wavelet coefficient of estimated signal required by soft threshold method possesses well continuity, but wavelet coefficient denoising in the front and back will produce constant deviation, so that reconstruction precision of signal descends. The estimated signals required by hard threshold method can produce additional vibration, so that smooth degree of reconstruction signal is lower. Therefore, the effect on signal denoising of these two methods isn't ideal.

Including the noise signal $f(t)=s(t)+n(t)$, denoising principle of wavelet transformation is that the wavelet coefficient formed by noise is eliminated with the greatest extent. The wavelet coefficient of estimated signal after denoising $\hat{w}_{j, k}$ is close to that of useful signal before denoising $w_{j, k}$. $\hat{w}_{j, k}$ located between ||$w_{j, k}-\lambda \|$ and $\left|w_{j, k}\right|$. Based on this thought, a new threshold function is put forward in the paper.

$$
\hat{w}_{j, k}=\left\{\operatorname{sgn}\left(w_{j, k}\right)\left[\left|w_{j, k}\right|-\left(1-\frac{1}{\exp \left(\frac{\alpha}{\left|w_{j, k}\right|}\right)}\right) \lambda\right],\left|w_{j, k}\right| \geq \lambda ;\right.
$$

In Formula $8, w_{j, k}$ represents wavelet coęfficient before denoising; $\lambda$ represents threshold value; $w_{j, k}$ represents wavelet coefficient after denoising; $\alpha$ is arbitrarily normal constant. When $\alpha$ is confirmed, Formula 9 can be a continuous and smooth state. Moreover, according to Formula 9, repression of including noise signal may be written as Formula 9:

$$
f(x)=\operatorname{sgn}(x)\left(|x|-\left(1-\frac{1}{\exp \left(\frac{\alpha}{\frac{|x|}{t}-t}\right)}\right) t\right),|x| \geq t
$$

Then,

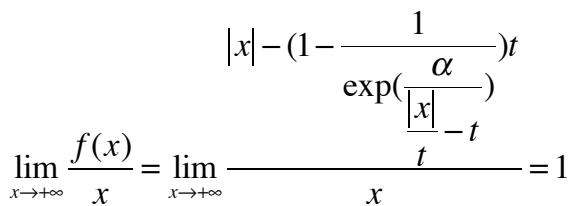

$\lim _{x \rightarrow-\infty} \frac{f(x)}{x}=-\lim _{x \rightarrow-\infty} \frac{|x|-\left(1-\frac{1}{\exp \left(\frac{\alpha}{|x|}\right)}\right) t}{x}=1$

$\lim _{x \rightarrow \infty}(f(x)-x)=0$ 


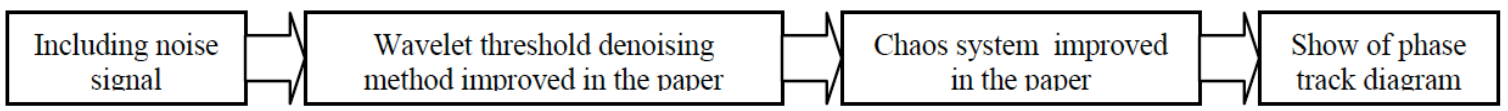

Fig. (3). Principle on common testing weak gas leakage signal by wavelet threshold denoising method and chaos duffing oscillator system.

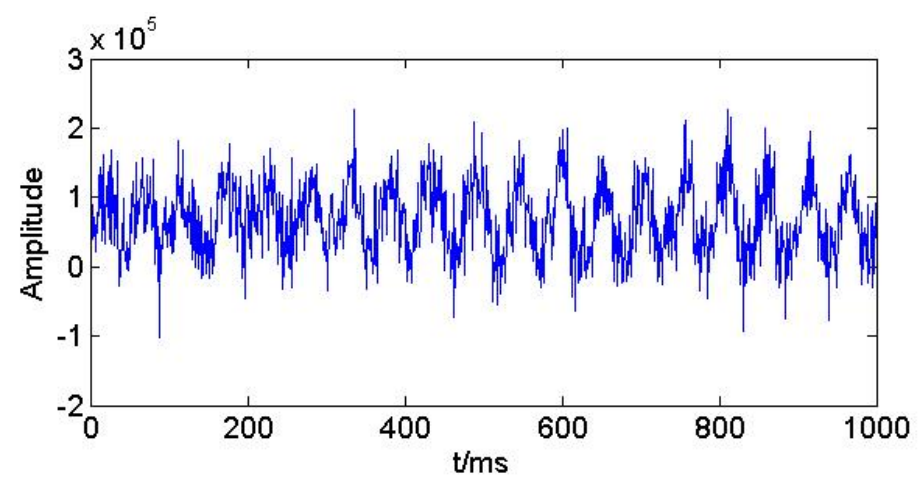

Fig. (4). Hardware sample signals of aircraft air-tightness cockpit required by ultrasonic testing instrument.

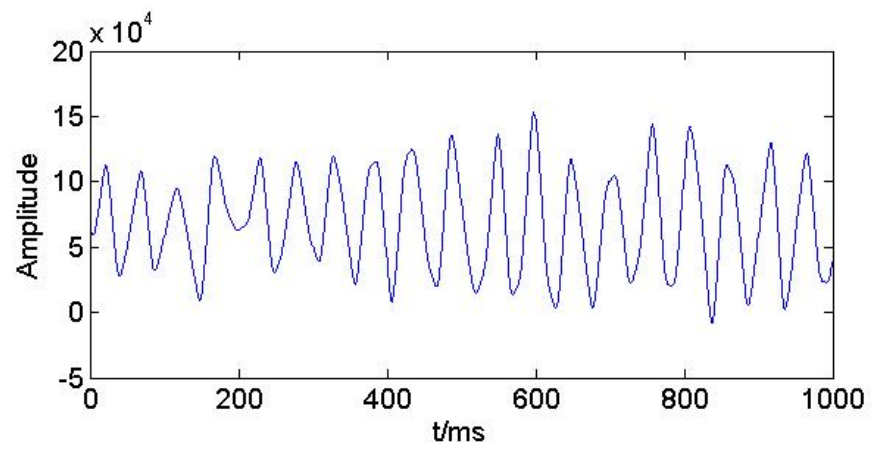

Fig. (5). Denoising effect of wavelet threshold denoising method improved in the paper.

The above-mentioned formula of deduction points out that with wavelet coefficient with increasing noise signal, the improved threshold function paper can reduce the deviation of wavelet coefficients denoising the front and back. Furthermore, if the range of parameter $\alpha$ is extended, then:

$\lim _{\alpha \rightarrow \infty} \operatorname{sgn}\left(w_{j, k}\left[\left|w_{j, k}\right|-\left(1-\frac{1}{\exp \left(\frac{\alpha}{\frac{\left|w_{j, k}\right|}{\lambda}-\lambda}\right)}\right) \lambda\right]=\operatorname{sgn}\left(w_{j, k}\right)\left(\left|w_{j, k}\right|-\lambda\right)\right.$

$\left.\lim _{\alpha \rightarrow 0} \operatorname{sgn}\left(w_{j, k}\right)\left[\left|w_{j, k}\right|-\left(1-\frac{1}{\exp \left(\frac{\alpha}{\frac{\left|w_{j, k}\right|}{\lambda}-\lambda}\right)}\right) \lambda\right]=\operatorname{sgn}\left(w_{j, k}\right) \mid w_{j, k}\right)$

That is to say, when $\alpha \rightarrow \infty$, Formula 9 has the same expression with that of the soft threshold function. When $\alpha \rightarrow 0$, Formula 9 has the same expression with that of the hard threshold function. Therefore, parameter $\alpha$ is properly regulated. Threshold function improved in the paper can not only keep smoothness of reconstruction signal, but also avoid constant deviation phenomenon.

\section{ANALYSIS OF PRACTICAL EXAMPLE}

In the work of gas leakage testing of aircraft air-tightness system, for eliminating electromagnetic interference noise in airport, we can not only rely on hardware denoising of ultrasonic testing instrument. So, at software level, we should utilize chaos Duffing oscillator equation and wavelet threshold denoising method improved in the paper, to acquire useful signal and eliminate noise to the maximum. According to this idea, the principle of signal discrimination and denoising is designed in Fig. (3).

\subsection{Improved Wavelet Threshold Denoising Method Deals with Practical Signal}

In an airport, gas leakage testing of one type of aircraft air-tightness cockpit is done under the strong noise background. Hardware sample signals required by ultrasonic instrument are shown in Fig. (4). From Fig. (4), it can be found that a useful signal is hardly observed because of strong noise interference.

By the use of wavelet threshold denoising method improved in the paper, hardware sample signals shown in Fig. (4) are denoised; the result is shown in Fig. (5).

Fig. (5) indicates that gas leakage signals of aircraft airtightness cockpit are better reconstructed by wavelet threshold denoising method improved in the paper. burr phenomena were applied by eliminating the noise. Barely singular 


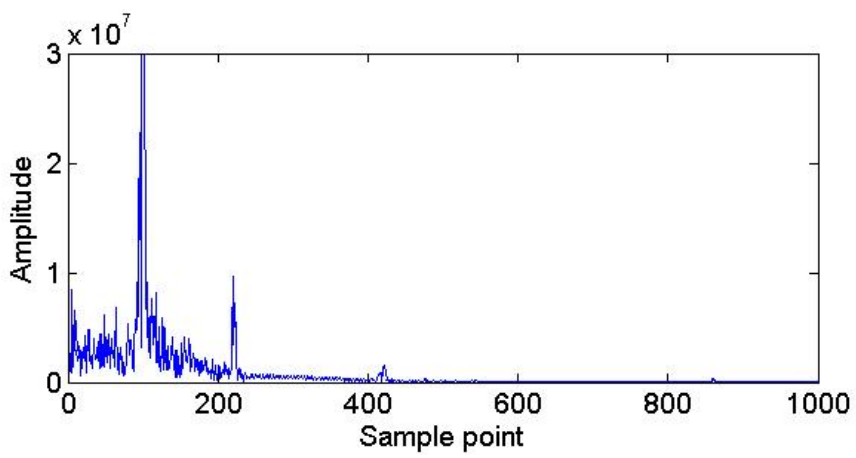

Fig. (6). Signal amplitude-frequency characteristic curve chart required by wavelet threshold denoising method improved in the paper.

Table 1. Comparison on denoising estimation indexes between hardware denoising and wavelet threshold denoising method improved in the paper.

\begin{tabular}{|c|c|c|}
\hline Denoising estimation indexes & Hardware denoising & wavelet threshold denoising method improved in the paper \\
\hline \hline Signal to noise ratio & -9.9033 & 2.6332 \\
\hline Root mean square error & 53412.6266 & 37450.1544 \\
\hline
\end{tabular}

points occur in the denoising signals. When denoising signals are analyzed from time domain to frequency domain, amplitude-frequency characteristic curve is obtained, as shown in Fig. (6).

Fig. (6) shows that the signal amplitude of the $100^{\text {th }}$ sample point is the strongest, after the $250^{\text {th }}$ sample point, signal amplitude approaches zero. According to the performance index of ultrasonic testing instrument used, the $100^{\text {th }}$ sample point is corresponding to $40 \mathrm{KHz}$ frequency signal, and the $250^{\text {th }}$ sample point is corresponding to $98 \mathrm{KHz}$ frequency signal. That means, more than $98 \mathrm{KHz}$ frequency signals have been wholly filtered, suggesting that wavelet threshold denoising method improved in the paper better eliminates high frequency noise in hardware sample signals. Besides, by the use of denoising estimation indexes, such as signal to noise ratio and root-mean-square error, denoising effect can be directly judged, as shown in Table 1 .

Table 1 reveals that in the strong noise background, compared with hardware denoising signals, denoising signals of wavelet threshold denoising method increased signal to noise ratio up to $12 \mathrm{db}$, and suppressed root-mean-square error up to 15962.4722. Thus, wavelet threshold denoising method improved in the paper possesses better capacity of denoising and extracting useful signals.

\subsection{Improved Chaos Duffing Oscillator System Deals with Practical Signal}

After hardware sample signals are denoised, signal frequency range will be limited between $10 \mathrm{KHz}$ and $100 \mathrm{KHz}$. For searching useful weak period signals of $40 \mathrm{KHz}$ frequency, chaos Duffing oscillator equation improved in the paper should be used for analysis.

Firstly, Duffing oscillator equation with $40 \mathrm{KHz}$ period driving force frequency is constructed, then, chaos system is regulated into the critical period state with no noise and no testing signals, as shown in Fig. (7).

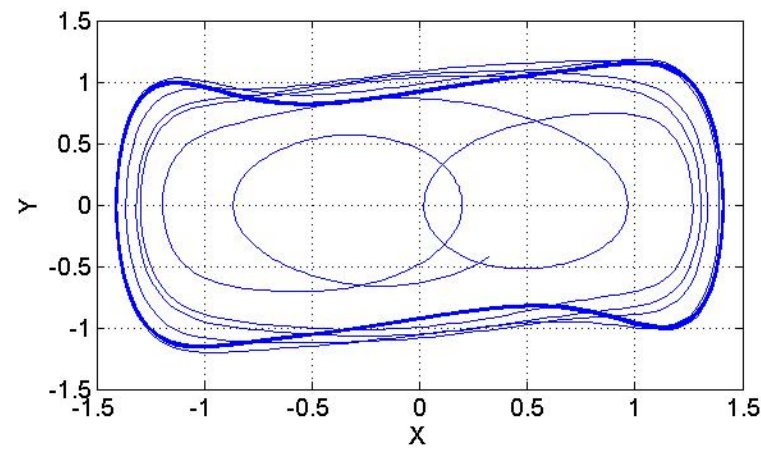

Fig. (7). Phase track diagram of chaos system with $40 \mathrm{khz}$ period driving force frequency, none noise and none testing signals.

At this time, by adding hardware sample signals into chaos system, owing to strong noise mixed in hardware sample signals, phase track state of chaos system will change. After front 80 sample points are added into the chaos system, the chaos system changes the critical period state into the chaos state. The result is shown in Fig. (8). After front 100 sample points are added into the chaos system, the system will obtain large scale period state. The result is shown in Fig. (9).

Fig. (8) and Fig. (9) show that the signal frequency of the $100^{\text {th }}$ sample point is equal to the period driving force frequency of chaos system. The sum of the signal amplitude of the $100^{\text {th }}$ sample point and amplitude of critical period driving force is bigger than chaos threshold of chaos system, so that chaos system obtains the large-scale periodic state. That means that the $100^{\text {th }}$ sample point reflects $40 \mathrm{KHz}$ frequency gas leakage signal, with gas leakage of the aircraft air-tightness cockpit. Therefore, according to state transformation of chaos system, the characteristics of useful gas leakage signal are to be further verified. 


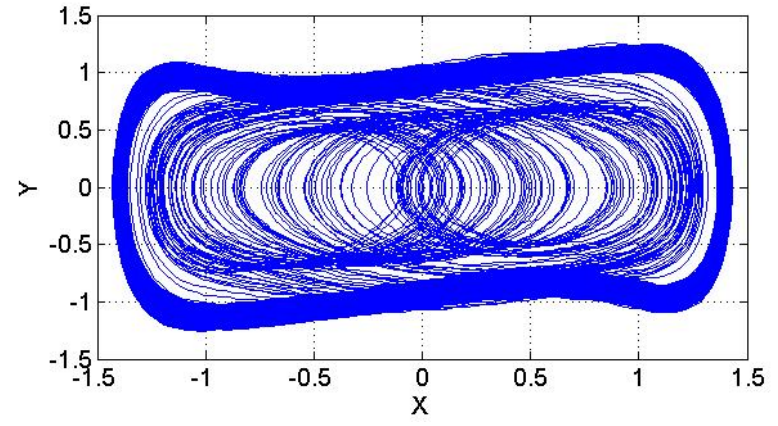

Fig. (8). After front 80 sample points are added, chaos system will be in chaos state.

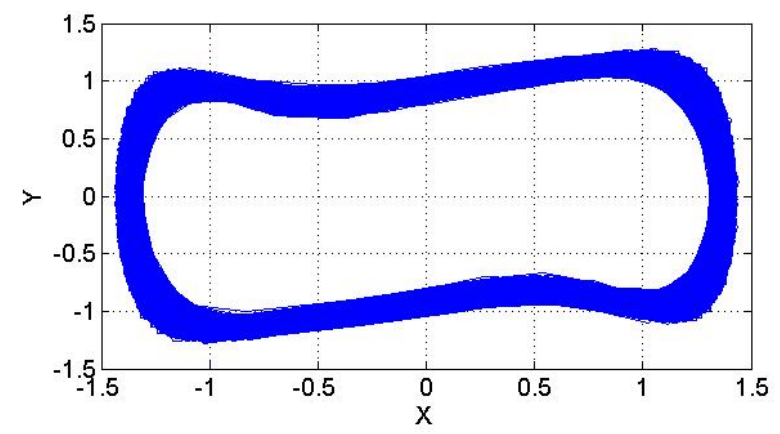

Fig. (9). After front 100 sample points are added, chaos system will be in large scale period state.

\section{CONCLUSION}

In the paper, testing and analysis of gas leakage signal of aircraft air-tightness cockpit in the strong noise background, were done based on four aspects. Firstly, according to ultrasonic signal characteristics of gas leakage, a mathematical model of ultrasonic testing gas leakage rate was deduced. Secondly, considering strong noise background and high frequency signal feature, traditional Duffing oscillator equation was improved, and the relationship between chaos threshold and noise standard deviation is mainly discussed. Thirdly, wavelet threshold denoising method was improved in the paper. Compared with Donoho soft and hard threshold denoising methods, the superiority of improved method in the paper is testified by formula analysis. Lastly, the effects of signal discrimination and denoising of improved chaos Duffing oscillator equation and improved wavelet threshold denoising method in the paper were inspected by practical examples.

\section{CONFLICT OF INTEREST}

The authors confirm that this article content has no conflict of interest.

\section{ACKNOWLEDGEMENTS}

Declared none.

\section{REFERENCES}

[1] G. Peng, C. Ji, and N. Ge, "Current status and future development of air tightness detection technique", Machine Tool \& Hydraulics, vol. 36 , no. 11 , pp. 172-174, 2008.

[2] D. Quan, "Study on a leakage test method in air-sealed experiments", Hydraulics Pneumatics \& Seals, vol. 6, pp. 25-27, 2005.

[3] H.X. Cai, Z. Dong, and C. Lin, "Research on air tightness detection method for aircraft gas system", Measurement \& Control Technology, vol. 31, pp. 9-11, 2012.

[4] Y.D. Wang, J.J. Zeng, and M.B. Tong, "Overview of several advances in aircraft system design", Advances in Aeronautical Science and Engineering, vol. 3, no. 2, pp. 144-149, 2012.

[5] Z.S. Gao, "Air Tight Cockpit Sealing Design", Aviation Industry Press: Beijing, 1982, pp. 13-15.

[6] W.X. Feng, Z.Q. Wang, and W.Y. Cheng, "Analysis of the nozzle model and hole model associated with high-pressure natural gas pipeline leakage", Safety and Environmental Engineering, vol. 16, no. 4, pp. 108-110, 2009.

[7] D.Y. Ma, P.Z. Li, and G.H. Dai, "The pressure relationship of turbulent flow noise", Acta Physica Sinica, vol. 27, no. 2, pp. 121125, 1978.

[8] D.Y. Ma, P.Z. Li, G.H. Dai, and H.Y. Wang, "Turbulent flow noise of high pressure jet", Acta Acustica, vol. 3, pp. 176-181, 1979.

[9] Y. Zhang, and G.Y. Ji, "Study of weak signal detection based on chaotic oscillator and wavelet theory", Electronic Measurement Technology, vol. 32, no. 6, pp. 40-43, 2009.

[10] G.Y. Wang, D.J. Chen, and J.Y. Lin, "The application of chaotic oscillatiors weak signal detection", IEEE Transactions on Industrial Electronics, vol. 46, no. 2, pp. 440-444, 2001.

[11] G.Y. Wang, and S. He, "A quantitative study on detection and weak signals by using chaotic duffing oscillators", IEEE Transactions on Circuits Systems, vol. 50, no. 7, pp. 945-953, 2003.

[12] L. Satish, and B. Nazneen, "Wavelet-based denoising of partial discharge signals buried in excessive noise and interference", IEEE Transaction on Dielectrics and Electrical Insulation, vol. 10, no. 2, pp. 367-375, 2003.

[13] Y.S Wang, W.Z. Jiang and J.J. Zhao, "A new method of weak signal detection using duffing oscillator and its simulation research", Acta Physica Sinica, vol. 57, no. 4, pp. 2053-2059, 2008.

[14] D.L. Donoho, and I.M. Johnstone, "Ideal spatial adaption via wavelet shrinkage", Biometrika, vol. 81, no. 12, pp. 425-455, 1994.

[15] D.L. Donoho, "De-noising by soft-thresholding", IEEE Transactions on IT, vol. 41, no. 3, pp. 617-643, 1995.

[16] D.L. Donoho, and I.M. Johnstone, "Adapting to unknown smoothness via wavelet shrinkage", Journal of American statistical Association, vol. 90, no. 432, pp. 1200-1224, 1995.

(C) Dawei and Jun; Licensee Bentham Open.

This is an open access article licensed under the terms of the (https://creativecommons.org/licenses/by/4.0/legalcode), which permits unrestricted, noncommercial use, distribution and reproduction in any medium, provided the work is properly cited. 